\title{
Biportal endoscopic discectomy for high-grade migrated lumbar disc herniation
}

\author{
Taewook Kang, MD, Si Young Park, MD, PhD, Gun Woo Park, MD, Soon Hyuck Lee, MD, PhD, \\ Jong Hoon Park, MD, PhD, and Seung Woo Suh, MD, PhD
}

Department of Orthopaedics, Korea University College of Medicine, Anam Hospital, Seoul, Korea

OBJECTIVE Although endoscopic procedures for lumbar disc herniation have improved greatly and offer many advantages, the indications are limited mostly to nonmigrated or low-grade migrated disc herniation. Endoscopic application in migrated disc herniation cases is still challenging and technically demanding. The goal in this study was to determine the feasibility of biportal endoscopic discectomy for removal of high-grade migrated disc herniation.

METHODS A retrospective review was performed in 262 patients who had undergone biportal endoscopic discectomy after the diagnosis of lumbar herniated disc. According to preoperative MRI findings, disc herniation was classified into 5 zones based on the direction and distance from the disc space. Patients were divided into 2 groups-a high-grade migration group and a low-grade migration group. Clinical outcomes were evaluated using the Oswestry Disability Index (ODI), visual analog scale (VAS), and modified Macnab criteria, and those outcomes and operation time were compared between the 2 groups.

RESULTS There were 10 patients with "high-grade up," 8 with "low-grade up," 98 with disc-level, 102 with "low-grade down," and 44 with "high-grade down" herniation, thereby yielding 54 patients in the high-grade group and 208 in the low-grade group. Demographic data for the 2 groups showed no significant difference. There was no significant difference between the 2 groups in ODI, VAS, and modified Macnab criteria. Operation time between the 2 groups was not significantly different (60.74 vs 65.63 minutes, $p>0.05)$.

CONCLUSIONS Biportal endoscopic discectomy can be effective for high-grade migrated lumbar disc herniation with no prolonged operation time and satisfactory clinical outcomes.

https://thejns.org/doi/abs/10.3171/2020.2.SPINE191452

KEYWORDS biportal endoscopic discectomy; disc herniation; high-grade migration; lumbar

$\mathrm{M}$ ICROENDOSCOPIC discectomy has been a common surgical procedure in patients with lumbar disc herniation. ${ }^{1}$ Percutaneous discectomy was first introduced by Hijikata in $1973 .{ }^{2}$ Kambin and Sampson introduced an endoscopic discectomy technique. ${ }^{3}$ Since then, new percutaneous endoscopic discectomy techniques have been developed. Minimally invasive spine surgery, which focuses on preservation of normal spine anatomy, is replacing conventional discectomy. ${ }^{4}$ Recently, percutaneous endoscopic lumbar discectomy is becoming widely used for the treatment of lumbar disc herniation. ${ }^{5}$ This procedure offers several advantages over conventional discectomy, such as less paravertebral muscle injury, bony structure preservation, and rapid recovery. ${ }^{6}$

The spectrum of endoscopic discectomy is expanding gradually, with some past contraindications now becoming indications. ${ }^{3,7,8}$ High-speed drills, curved forceps, and probes have enabled the treatment of various types of lumbar disc herniation and lumbar spinal stenosis.,10
Although successful outcomes have been reported with improvements in techniques and instruments, migrated disc herniations remain challenging. ${ }^{1}$ Migrated disc herniations are still regarded as technically demanding because the procedure is performed through a small working space. Also, there is suspicion among surgeons regarding whether endoscopic discectomy can effectively remove migrated disc fragments. Migrated disc herniations with sequestration require additional resection of the lamina, which may alter normal spinal segment biomechanics, and this can worsen back pain and increase postoperative instability. ${ }^{11}$ Percutaneous endoscopic discectomy offers definite advantages, but the indications have mainly been limited to nonmigrated or low-grade migrated herniations. The failure of this treatment is mainly due to insufficient decompression because there are more remnant fragments in patients with high-grade migrated disc herniations. ${ }^{11}$ Therefore, for high-grade migrated disc cases, conventional discectomy is recommended. ${ }^{5,12}$ 


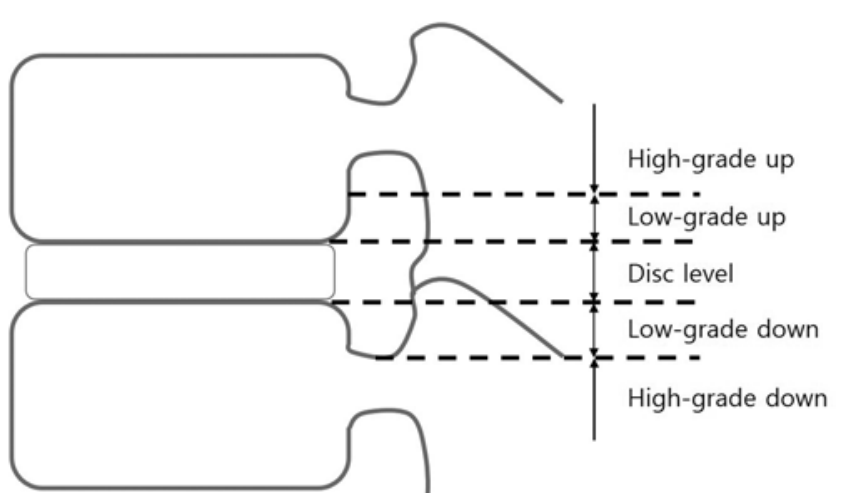

FIG. 1. Schematic representation of disc herniation. The direction and degree of migration of herniated discs is divided into 5 zones (high-grade up, low-grade up, disc level, low-grade down, and high-grade down).

Biportal endoscopy is an emerging technique among various minimally invasive spinal surgery options. Biportal endoscopic spinal surgery offers several advantages with minimal limitations. Sufficient decompression can be achieved with free handling of the instruments under magnified clear view, and several studies have reported satisfactory results after treatment with biportal endoscopic spinal surgery in lumbar disc herniations and lumbar spinal stenosis. ${ }^{10,13,14}$ Biportal endoscopic discectomy has shown favorable clinical outcomes and minimal blood loss, reduced length of hospital stay, and reduced postoperative pain compared with microendoscopic discectomy. ${ }^{15}$

Endoscopic application in high-grade migrated lumbar disc herniation cases is still challenging and technically demanding. The purpose of this study was to investigate the feasibility of biportal endoscopic discectomy for removal of high-grade migrated lumbar disc herniation.

\section{Methods}

\section{Study Design and Participants}

A retrospective review was performed in patients who had undergone biportal endoscopic discectomy by a single surgeon after diagnosis of lumbar disc herniation between January 2016 and June 2018. This study was approved by our institutional review board. Because this study reviewed preexisting data, informed consent was waived.

We hypothesized that clinical outcomes and operation time after biportal endoscopic discectomy in the highgrade migration group would be comparable to those in the low-grade migration group. For subgroup analysis, we also compared outcomes of "high-grade up" and "highgrade down" migration.

The inclusion criteria were as follows: 1) neurological symptoms (radiculopathy, sensory changes, motor weakness) due to single-level lumbar disc herniation; 2) symptoms correspond to preoperative MRI; 3) unsuccessful conservative treatment for at least 4 weeks; 4) no history of previous spinal surgery; and 5) age 18-65 years. The exclusion criteria were as follows: 1) lumbar spinal stenosis confirmed by MRI; 2) segmental instability confirmed by dynamic radiographs; 3 ) recurrent disc herniation; and 4) infection.

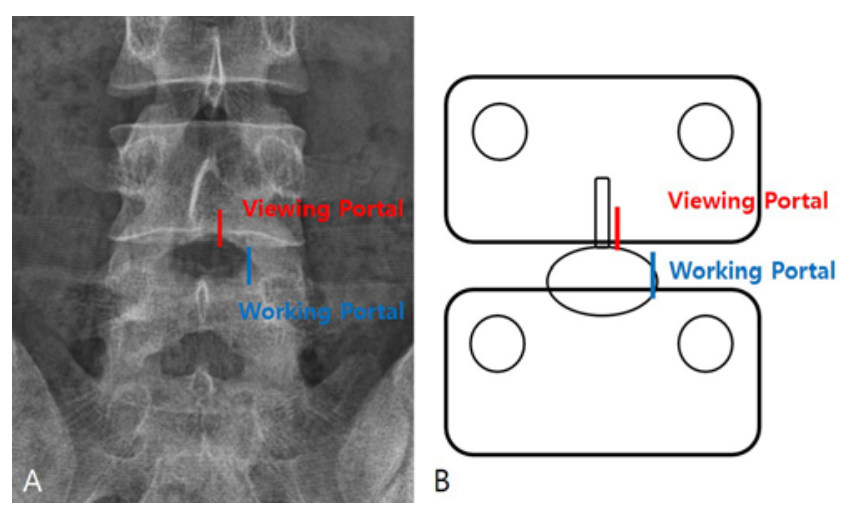

FIG. 2. Portal placement in biportal endoscopic spinal surgery, right side. Portal locations on the anteroposterior radiograph $(\mathbf{A})$ and on the schematic view (B). Figure is available in color online only.

Disc herniation was classified into 5 zones according to preoperative MRI findings. Depending on the direction and distance from the disc space, 5 groups were formed, as follows: high-grade up; low-grade up; disc level; highgrade down; and low-grade down. If the distance of migration is above the lower half of the inferior pedicle, highgrade up migration is defined. If the distance of migration is between the upper margin of disc space and the upper half of the inferior pedicle, low-grade up migration is defined. If the distance of migration is between the lower margin of the disc space and superior pedicle, low-grade down migration is defined. If the distance of the migration is below the superior pedicle, high-grade down migration is defined (Fig. 1). Ultimately, patients were divided into a high-grade migration group and a low-grade migration or nonmigration (i.e., disc level) group.

\section{Surgical Procedures}

All patients underwent surgery in a prone position under general anesthesia. Two separate oblique 1-cm skin incisions were made above and below the disc space (Fig. 2). The viewing portal was made cranially, and a $0^{\circ}$ arthroscope was inserted. A saline irrigation pump was connected to the viewing portal and set to a pressure of $30 \mathrm{~mm}$ $\mathrm{Hg}$. Continuous flow of saline perfusion was essential to prevent excessive elevation of the epidural pressure, which can cause elevation of intracranial pressure. The working portal was made caudally, and conventional spinal surgical instruments and arthroscopic instruments were inserted through the working portal. Hemilaminectomy was performed using a burr and the Kerrison rongeur until the upper edge of the deep portion of the ligamentum flavum was exposed. The ligamentum flavum was carefully dissected and completely resected. An incision was made on the disc, and pituitary forceps and curettes were used to perform discectomy and remove fragments of the herniated disc. The procedure was completed after confirming the decompression status and freely moving exiting nerve root with a ball tip probe (Video 1).

VIDEO 1. Removal of high-grade up-migrated disc herniation using biportal endoscopic spinal surgery. Copyright Taewook Kang.

Published with permission. Click here to view.

Using a $30^{\circ}$ arthroscope would be helpful by allowing a 
Kang et al.

TABLE 1. Demographic data in 262 patients with migrated lumbar disc herniation

\begin{tabular}{cccc}
\hline Variable & Low Grade $(\mathrm{n}=208)$ & High Grade $(\mathrm{n}=54)$ & $\mathrm{p}$ Value \\
\hline Age $(\mathrm{yrs})$ & 50.72 & 53.74 & 0.599 \\
\hline Sex $(\mathrm{M} / \mathrm{F})$ & $106 / 102$ & $30 / 24$ & 0.672 \\
\hline BMl $\left(\mathrm{kg} / \mathrm{m}^{2}\right)$ & 25.28 & 23.89 & 0.207 \\
\hline Op level & & & 0.115 \\
\hline L3/4 & 8 & 8 & \\
\hline L4/5 & 134 & 34 & \\
\hline L5/S1 & 66 & 12 & \\
\hline
\end{tabular}

wider view of the surgical field and direct visualization of migrated disc fragments. A Hemovac drain is placed in all patients through the working portal to prevent hematoma formation.

\section{Outcome Measures}

All patients completed self-assessment questionnaires and provided demographic and clinical information.

Operation time, length of hospital stay, and Hemovac drain output were compared between the 2 groups. Postoperative MRI was obtained 2 weeks after surgery, and 2 orthopedic surgeons and 1 radiologist interpreted the imaging. Clinical outcomes were assessed with visual analog scale (VAS), Oswestry Disability Index (ODI), and modified Macnab criteria. Clinical follow-ups were performed at the end of $1,3,6$, and 12 months. The intensity of leg pain was measured by VAS from no pain (point 0 ) to worst pain imaginable (point 10). The clinical outcome was evaluated by an independent surgeon using the modified Macnab criteria.

\section{Statistical Analysis}

Differences in mean VAS and ODI scores between baseline and each postoperative period were assessed by paired t-tests. Demographic data, predetermined clinical data, and the incidence of adverse events were compared using the Mann-Whitney U-test and Fisher's exact test. The level of significance was set at $p<0.05$. All statistical analyses were performed using SPSS version 20.0 (IBM Corp.).

\section{Results}

\section{Demographic Data}

A total of 271 patients were assessed for eligibility, and 9 patients did not meet our inclusion criteria. Age, sex, body mass index (BMI), and operative level were not significantly different between the 2 groups (Table 1). All patients were followed up for more than 12 months after surgery. The mean follow-up period was $16 \pm 2.5$ months (mean $\pm \mathrm{SD}$ ). The mean age of high-grade group patients was 53.74 years and that of the low-grade group was 50.72 years $(\mathrm{p}=0.599)$. The mean BMI of the high-grade group was $23.89 \mathrm{~kg} / \mathrm{m}^{2}$ and that of the low-grade group was $25.28 \mathrm{~kg} / \mathrm{m}^{2}(\mathrm{p}=0.207)$. The operative level was $\mathrm{L} 3 / 4$ in 8 patients, L4/5 in 34 patients, and L5/S1 in 12 patients in the high-grade group; and it was L3/4 in 8 patients, L4/5

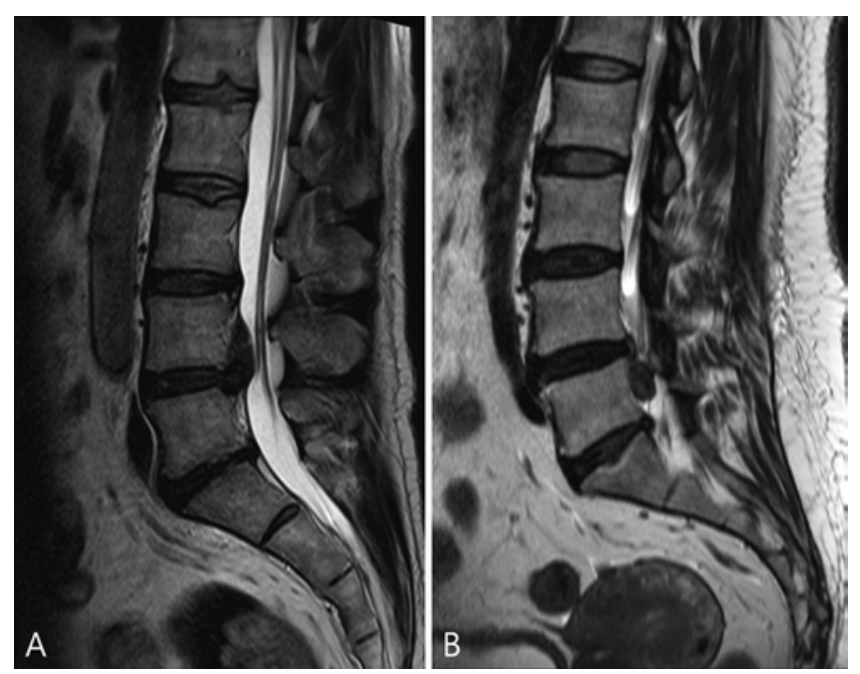

FIG. 3. Preoperative MR images of high-grade migrated discs. A: Highgrade up migration. B: High-grade down migration.

in 134 patients, and L5/S1 in 66 patients in the low-grade group $(\mathrm{p}=0.115)$.

There were 10 patients with high-grade up, 8 with lowgrade up, 98 with disc-level, 102 with low-grade down, and 44 with high-grade down herniation (Fig. 3). Altogether, 54 patients in the high-grade group and 208 in the low-grade group were evaluated (Fig. 4).

\section{Clinical Data}

Ninety-eight percent of patients (258/262) underwent postoperative MRI at 2 weeks after surgery. Postoperative MRI of the patients in both groups showed well-decompressed status after surgery (Fig. 5).

The average operation time between the 2 groups was not significantly different (65.63 vs 60.74 minutes, $\mathrm{p}=$ 0.245 ). Length of hospital stay between the 2 groups was not significantly different (3.48 vs 3.65 days, $\mathrm{p}=0.557$ ). Hemovac drain output between the 2 groups was also not significantly different ( 37.78 vs $42.07 \mathrm{ml}, \mathrm{p}=0.556$ ). Only 1 patient in the low-grade group had hematoma, and no complication was identified in the high-grade group. No patient in either group had a dural tear or CSF leakage. Three patients in the low-grade group underwent reoperation performed using the biportal endoscopic technique due to recurring symptoms, and no revision surgery was performed in the high-grade group (Fig. 6). Clinical outcomes assessed using VAS and ODI scores and modified Macnab criteria showed good results in both groups, with no significant statistical difference (Table 2).

\section{Subgroup Analysis}

Ten patients in the high-grade up group and 44 in the high-grade down group were evaluated. Operation time, hospital stay, and Hemovac output were not significantly different between the 2 groups (Table 3). The average operation times between the 2 groups were not significantly different (57.5 vs 58.86 minutes, $\mathrm{p}=0.89$ ). Length of hospital stay between the 2 groups was not significantly different (3.8 vs 3.0 days, $p=0.487$ ). Hemovac drain output 


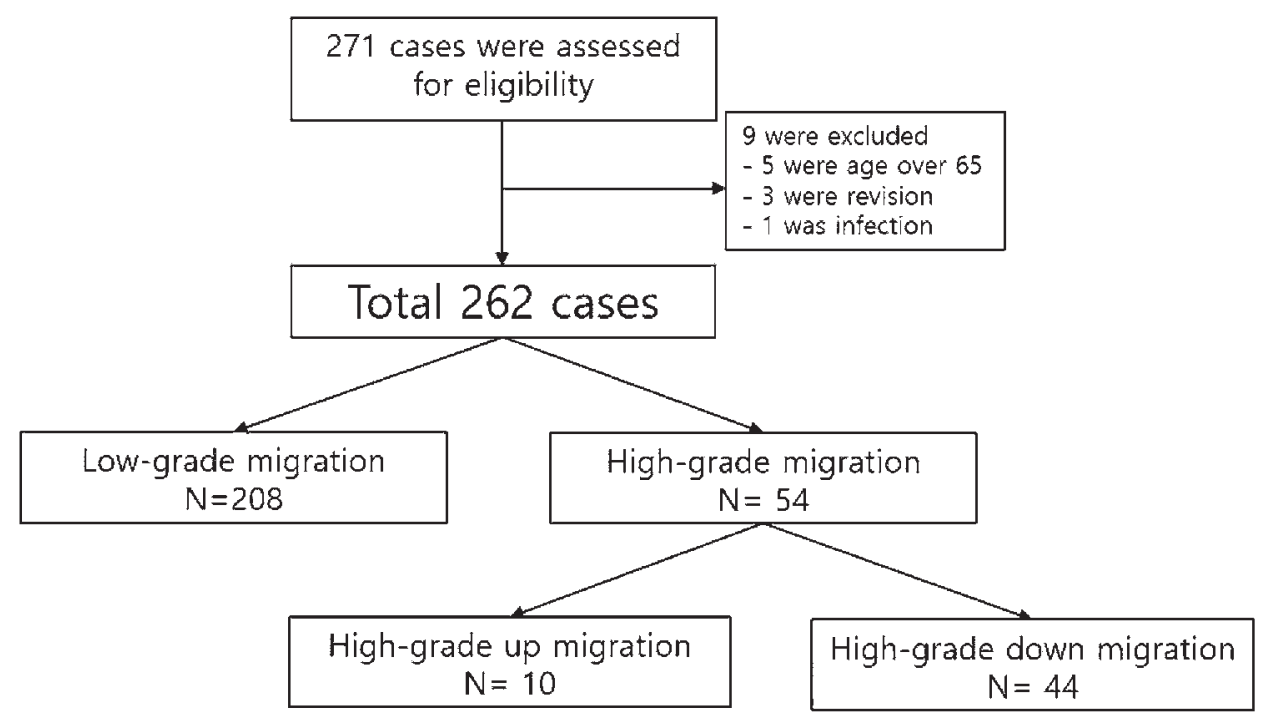

FIG. 4. Flowchart showing the trial profile.

between the 2 groups was also not significantly different (29.0 vs $42.07 \mathrm{ml}, \mathrm{p}=0.208$ ). No complication was found and no revision surgery was performed in either group.

Satisfactory results were obtained in all cases in both groups. There was no significant difference between the 2 groups regarding ODI and VAS scores.

\section{Discussion}

In this study, biportal endoscopic discectomy showed satisfactory clinical outcomes in high-grade migrated disc
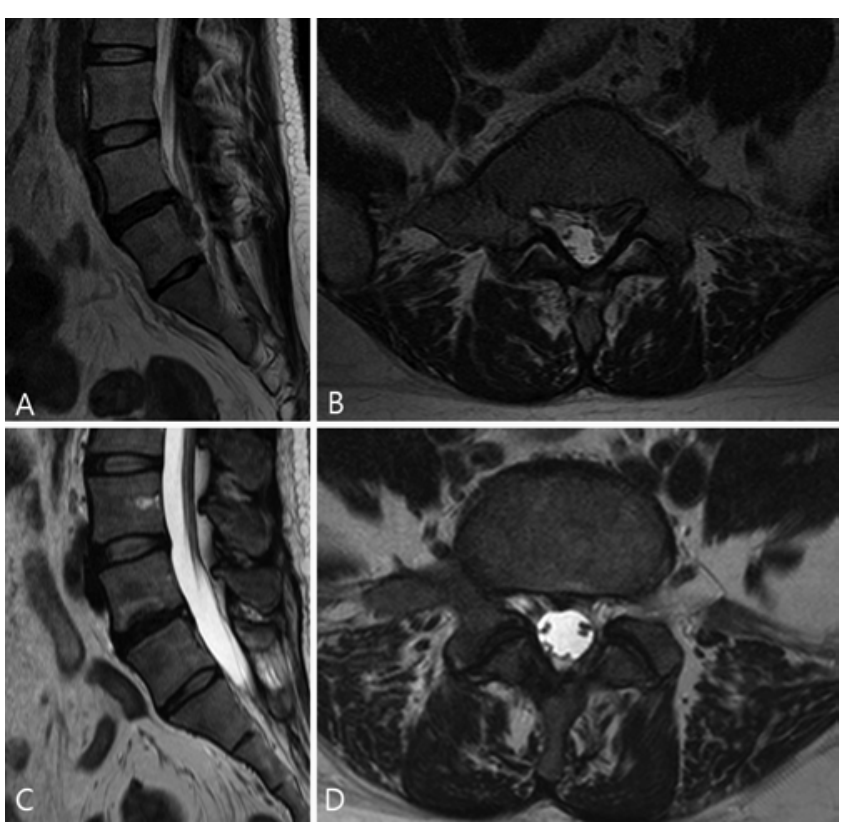

FIG. 5. High-grade down migration at the L5/S1 level. A: Preoperative sagittal MR image. B: Preoperative axial MR image. C: Well-decompressed status on postoperative sagittal MR image after 6 months. D: Well-decompressed status on postoperative axial MR image after 6 months. herniations with similar operation time. Microendoscopic surgery in which the tubular retractor system introduced by Foley and Smith is used has been the gold standard for lumbar discectomy. ${ }^{16}$ Recently, several studies related to endoscopic spinal surgery have been reported and the results are improving. ${ }^{5,8,17}$ Endoscopic spinal surgery has several advantages over conventional surgery, and one of the main advantages for patients is a faster return to work. Furthermore, by reducing the possibility of excessive resection of bones and ligaments, postoperative iatrogenic instabilities can be prevented..$^{18}$ The development of instrumentation and techniques has expanded the indications for endoscopic surgery. Endoscopic discectomy provides results comparable to conventional techniques while preserving normal anatomy. ${ }^{7,8}$

Disc fragment migration occurs frequently. When a fragment enters the anterior epidural space through the posterior longitudinal ligament, it migrates in 35\%-72\% of cases. ${ }^{19,20}$ Disc fragments can migrate up to the midlevel of the vertebra and can be further separated into multiple fragments. Therefore, there is a greater risk of missing fragments in high-grade migrated discs. ${ }^{21}$ Removal of migrated disc fragments frequently requires extensive bone resection, including lamina, pars interarticularis, and even facet joints, which may result in postoperative instability. Migrated disc fragments have been considered inaccessible with endoscopic spinal surgery, and this technique does not guarantee complete removal, because of rigid instrumentation, limited visualization, and inability to reach and grasp migrated disc fragments. ${ }^{6}$ Most surgeons would suggest that migrated disc fragments cannot be removed safely with endoscopic spinal surgery. ${ }^{5}$

Some authors have reported the anatomical classification of migrated disc herniation depending on the direction and distance from the disc space, and they have recommended open surgery for high-grade migrated disc herniation. ${ }^{5}$ Microendoscopic discectomy is currently the standard treatment for lumbar disc herniations. As a minimally invasive surgery, percutaneous endoscopic disc- 


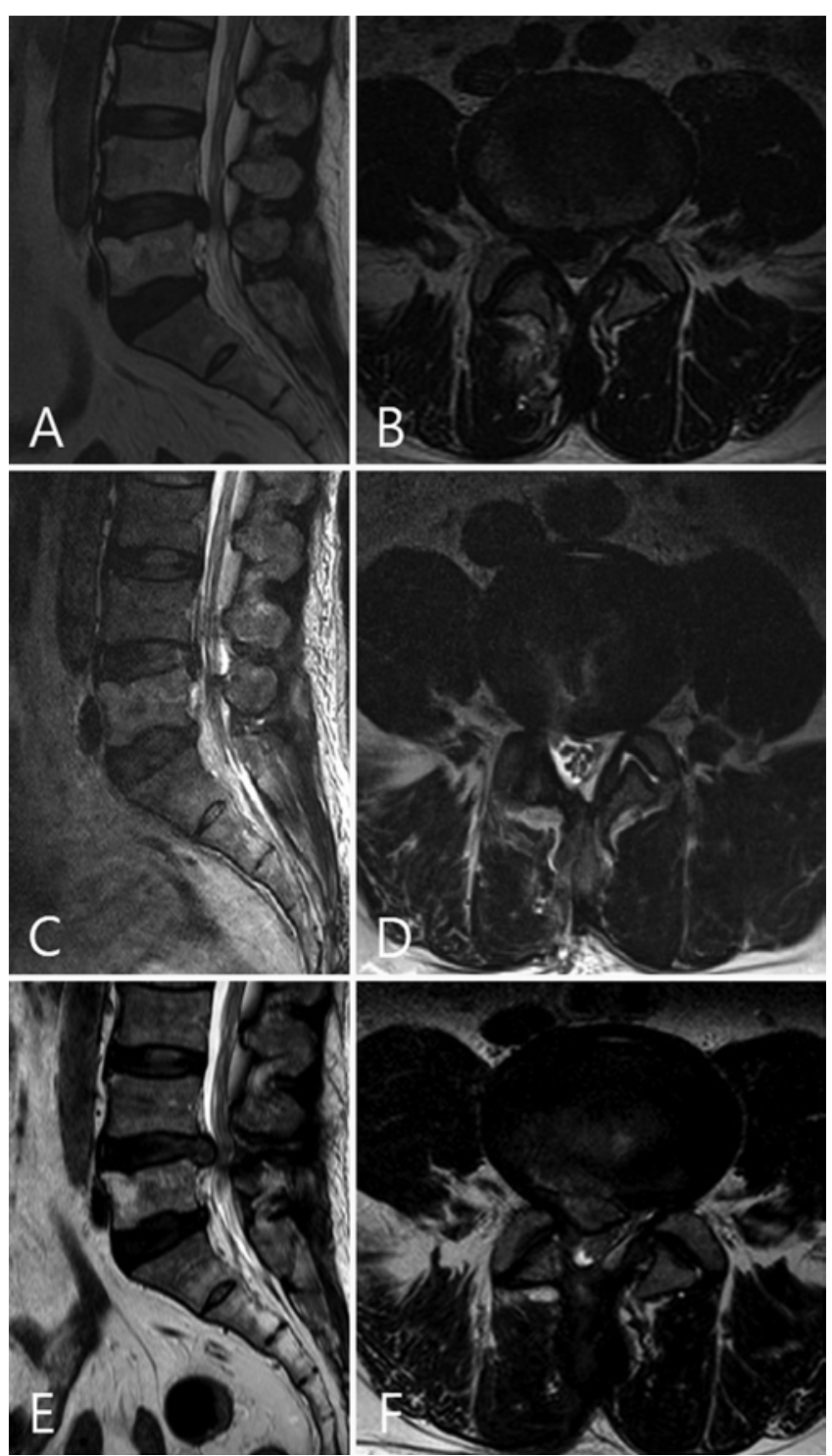

FIG. 6. Low-grade down migration at $L 4 / 5$ level: revision case. A: Preoperative sagittal MR image. B: Preoperative axial MR image. C: Welldecompressed status on postoperative sagittal MR image after 1 month. D: Well-decompressed status on postoperative axial MR image after 1 month. E: Sagittal MR image obtained 3 months postoperatively showing that herniation recurred at the same level. F: Axial MR image obtained 3 months postoperatively showing that herniation recurred at the same level.

ectomy is becoming more popular. However, high-grade migrated disc herniations are technically demanding and, due to the high failure rate, open microdiscectomy is often recommended. Extensive resection of the lamina is needed with the interlaminar approach, but there is a limitation of enough bone resection even with the improvement of surgical instruments. High-grade migrated disc herniations still remain challenging and technically demanding.

Compared with these, our study has fewer limitations. Biportal endoscopic surgery showed similar clinical outcomes compared with microendoscopic surgery, but it also showed improved blood loss, reduced length of hospital stay, and reduced postoperative pain. ${ }^{10,15}$ It allows
TABLE 2. Clinical data in 262 patients with migrated lumbar disc herniation

\begin{tabular}{|c|c|c|c|}
\hline Variable & $\begin{array}{l}\text { Low Grade } \\
(n=208)\end{array}$ & $\begin{array}{l}\text { High Grade } \\
(n=54)\end{array}$ & $\begin{array}{c}p \\
\text { Value }\end{array}$ \\
\hline Op time (mins) & $65.63 \pm 16.5$ & $60.74 \pm 18.1$ & 0.245 \\
\hline Hospital stay (days) & $3.48 \pm 2.8$ & $3.65 \pm 2.5$ & 0.557 \\
\hline Hemovac output (ml) & $37.78 \pm 5.2$ & $42.07 \pm 6.0$ & 0.556 \\
\hline No. of complications & 1 & 0 & \\
\hline No. of revisions & 3 & 0 & \\
\hline VAS score & & & NS \\
\hline Preop & $8.4 \pm 1.3$ & $8.2 \pm 1.2$ & \\
\hline Immediately postop & $6.5 \pm 0.9$ & $6.3 \pm 1.0$ & \\
\hline 1 mo postop & $3.5 \pm 1.1$ & $2.6 \pm 1.0$ & \\
\hline 3 mos postop & $2.3 \pm 0.8$ & $2.2 \pm 0.8$ & \\
\hline 6 mos postop & $2.1 \pm 0.9$ & $1.9 \pm 0.8$ & \\
\hline 1 yr postop & $1.4 \pm 0.7$ & $1.5 \pm 0.7$ & \\
\hline ODI & & & NS \\
\hline Preop & $42 \pm 5.1$ & $41 \pm 5.5$ & \\
\hline Immediately postop & $31 \pm 3.3$ & $32 \pm 3.7$ & \\
\hline 1 mo postop & $21 \pm 3.1$ & $17 \pm 3.5$ & \\
\hline 3 mos postop & $11 \pm 2.6$ & $12 \pm 2.8$ & \\
\hline 6 mos postop & $11 \pm 2.5$ & $11 \pm 2.5$ & \\
\hline 1 yr postop & $9 \pm 2.2$ & $10 \pm 2.5$ & \\
\hline Modified Macnab criteria & & & NS \\
\hline Excellent & 153 & 31 & \\
\hline Good & 55 & 23 & \\
\hline
\end{tabular}

NS $=$ not significant.

Except for the number of patients with complications, revisions, and excellent or good Macnab results, values are expressed as the mean \pm SD.

wider vision with high magnification because the distance between the surgeon's eye and the lesion is close. Ordinary arthroscopic and spine instruments were used without the need for special endoscopic instruments. By using 2 portals, free movement, handling, and angulation of the surgical instruments and the arthroscope are allowed independent of each other without crowding of instruments. In addition, this technique provides technical flexibility with sufficient bony and soft-tissue work, comparable to conventional surgery. Continuous saline perfusion can control bleeding and reduce the risk of infection, and the risk of a dural tear is reduced by slight compression of the dura mater by continuous saline perfusion. Some past contraindications are now the indications of the present., ${ }^{7,10}$

TABLE 3. Subgroup analysis of 54 patients with high-grade migrated lumbar disc herniation

\begin{tabular}{lccc}
\hline \multicolumn{1}{c}{ Variable } & $\begin{array}{c}\text { High Grade Up } \\
(\mathrm{n}=10)\end{array}$ & $\begin{array}{c}\text { High Grade Down } \\
(\mathrm{n}=44)\end{array}$ & $\begin{array}{c}\mathrm{p} \\
\text { Value }\end{array}$ \\
\hline Op time (mins) & $57.50 \pm 18.22$ & $58.86 \pm 17.57$ & 0.89 \\
\hline Hospital stay (days) & $3.8 \pm 2.1$ & $3.0 \pm 2.0$ & 0.487 \\
\hline Hemovac output (ml) & $29.0 \pm 4.1$ & $42.07 \pm 3.7$ & 0.208 \\
\hline
\end{tabular}

Values are expressed as the mean $\pm S D$. 
However, there are concerns about increased intracranial pressure due to the large amount of irrigation fluid used during biportal endoscopic surgery.

In our study, the high-grade migration group showed almost the same operation time as the low-grade migration group with no significant difference. A magnified clear view, free movement of instruments, and bony work performed using a burr enabled an easier approach and removal of high-grade migrated discs.

This study had several limitations. First, a retrospective medical record analysis was performed. Second, we examined a small number of patients. However, there has been no study about high-grade migration with a reasonable number of patients treated by biportal endoscopic surgery. The feasibility and benefits of biportal endoscopic discectomy compared with microendoscopic discectomy for high-grade lumbar disc herniation should be evaluated in a larger patient population with a prospectively controlled design.

\section{Conclusions}

Biportal endoscopic discectomy showed satisfactory clinical outcomes without longer operation times in patients with high-grade migrated lumbar disc herniation. Therefore, biportal endoscopic spinal surgery can be an effective treatment for high-grade migrated lumbar disc herniation.

\section{References}

1. Choi KC, Lee DC, Shim HK, et al. A strategy of percutaneous endoscopic lumbar discectomy for migrated disc herniation. World Neurosurg. 2017;99:259-266.

2. Hijikata S, Yamagishi M, Nakayama T, et al. Percutaneous discectomy: a new treatment method for lumbar disc herniation. J Toden Hosp. 1975;5:5-13.

3. Kambin P, Sampson S. Posterolateral percutaneous suctionexcision of herniated lumbar intervertebral discs. Report of interim results. Clin Orthop Relat Res. 1986;(207):37-43.

4. Choi G, Prada N, Modi HN, et al. Percutaneous endoscopic lumbar herniectomy for high-grade down-migrated L4-L5 disc through an L5-S1 interlaminar approach: a technical note. Minim Invasive Neurosurg. 2010;53(3):147-152.

5. Lee S, Kim SK, Lee SH, et al. Percutaneous endoscopic lumbar discectomy for migrated disc herniation: classification of disc migration and surgical approaches. Eur Spine J. 2007;16(3):431-437.

6. Choi KC, Lee JH, Kim JS, et al. Unsuccessful percutaneous endoscopic lumbar discectomy: a single-center experience of 10,228 cases. Neurosurgery. 2015;76(4):372-381.

7. Choi KC, Kim JS, Park CK. Percutaneous endoscopic lumbar discectomy as an alternative to open lumbar microdiscectomy for large lumbar disc herniation. Pain Physician. 2016;19(2):E291-E300.

8. Ruetten S, Komp M, Merk H, Godolias G. Full-endoscopic interlaminar and transforaminal lumbar discectomy versus conventional microsurgical technique: a prospective, randomized, controlled study. Spine (Phila Pa 1976). 2008;33(9):931-939.

9. Ahn Y, Oh HK, Kim H, et al. Percutaneous endoscopic lumbar foraminotomy: an advanced surgical technique and clinical outcomes. Neurosurgery. 2014;75(2):124-133.

10. Kang T, Park SY, Kang CH, et al. Is biportal technique/ endoscopic spinal surgery satisfactory for lumbar spinal ste- nosis patients?: a prospective randomized comparative study. Medicine (Baltimore). 2019;98(18):e15451.

11. Osman SG, Nibu K, Panjabi MM, et al. Transforaminal and posterior decompressions of the lumbar spine. A comparative study of stability and intervertebral foramen area. Spine (Phila Pa 1976). 1997;22(15):1690-1695.

12. Lee SH, Kang BU, Ahn Y, et al. Operative failure of percutaneous endoscopic lumbar discectomy: a radiologic analysis of 55 cases. Spine (Phila Pa 1976). 2006;31(10):E285-E290.

13. Choi DJ, Jung JT, Lee SJ, et al. Biportal endoscopic spinal surgery for recurrent lumbar disc herniations. Clin Orthop Surg. 2016;8(3):325-329.

14. Moon ASM, Rajaram Manoharan SR. Endoscopic spine surgery: current state of art and the future perspective. Asian Spine J. 2018;12(1):1-2.

15. Kim SK, Kang SS, Hong YH, et al. Clinical comparison of unilateral biportal endoscopic technique versus open microdiscectomy for single-level lumbar discectomy: a multicenter, retrospective analysis. J Orthop Surg Res. 2018;13(1):22.

16. Perez-Cruet MJ, Foley KT, Isaacs RE, et al. Microendoscopic lumbar discectomy: technical note. Neurosurgery. 2002;51(5) (suppl):S129-S136.

17. Ditsworth DA. Endoscopic transforaminal lumbar discectomy and reconfiguration: a postero-lateral approach into the spinal canal. Surg Neurol. 1998;49(6):588-598.

18. Yeung AT, Tsou PM. Posterolateral endoscopic excision for lumbar disc herniation: surgical technique, outcome, and complications in 307 consecutive cases. Spine (Phila Pa 1976). 2002;27(7):722-731.

19. Ebeling U, Reulen HJ. Are there typical localisations of lumbar disc herniations? A prospective study. Acta Neurochir (Wien). 1992;117(3-4):143-148.

20. Fries JW, Abodeely DA, Vijungco JG, et al. Computed tomography of herniated and extruded nucleus pulposus. $J$ Comput Assist Tomogr. 1982;6(5):874-887.

21. Choi G, Lee SH, Lokhande P, et al. Percutaneous endoscopic approach for highly migrated intracanal disc herniations by foraminoplastic technique using rigid working channel endoscope. Spine (Phila Pa 1976). 2008;33(15):E508-E515.

\section{Disclosures}

The authors report no conflict of interest concerning the materials or methods used in this study or the findings specified in this paper.

\section{Author Contributions}

Conception and design: SY Park, Kang. Acquisition of data: Kang, GW Park. Analysis and interpretation of data: Kang. Drafting the article: Kang. Critically revising the article: Lee. Reviewed submitted version of manuscript: Lee, Suh. Statistical analysis: GW Park. Administrative/technical/material support: JH Park, Suh. Study supervision: Lee, JH Park, Suh.

\section{Supplemental Information \\ Videos}

Video 1. https://vimeo.com/398799221.

\section{Previous Presentations}

Presented at the Asia Pacific Spine Society meeting, April 4-6, 2019, Incheon, Korea.

\section{Correspondence}

Si Young Park: Korea University College of Medicine, Anam Hospital, Seoul, Korea. drspine90@kumc.or.kr. 